\title{
El papel de las emociones en la enseñanza intercultural del español como lengua extranjera (ELE)
}

\section{The role of emotions in intercultural teaching of Spanish as a foreign language}

\author{
Małgorzata Spychała-Wawrzyniak \\ Uniwersytet im. Adama Mickiewicza, Poznań \\ malgorzata.spychala@amu.edu.pl
}

\begin{abstract}
Emotions, both positive and negative, are part of our lives. However, until the 1960s, little attention was paid to the role they played in learning foreign languages. It all changed with the development of humanistic psychology, which focused, among others, on the integration of cognitive and affective teaching. Emotions also accompany encounters of individuals representing two different cultures. Intercultural communication is a complex process, which often involves culture shock, conflicts and various types of interpersonal relations. The aim of this paper is to accentuate the role of emotions in foreign language teaching. We will put forward several proposals concerning affective learning viewed from the perspective of intercultural communication. Finally, we will present the results of the study conducted among learners of Spanish, which illustrates the importance of affective factors in constructing students' positive attitudes towards language and culture.
\end{abstract}

Keywords: emotions, affect in language learning, neurodidactics, intercultural communications, Spanish learners

\section{LAS EMOCIONES Y LA ENSEÑANZA INTERCULTURAL}

Hasta los años sesenta se prestaba poca atención a la importancia de los factores emocionales en el aprendizaje de lenguas. Todo cambió con el desarrollo de la psicología humanística, que se centra en la necesidad de unir la enseñanza cognitiva con la afectiva. A partir de esta, los nuevos métodos que surgieron, entre ellos el 
método directo o el enfoque comunicativo, empezaron a promover la enseñanza afectiva teniendo en cuenta la comunicación intercultural.

El encuentro de personas pertenecientes a dos culturas diferentes es un acto muy complejo, que está estrechamente vinculado con distintos tipos de relaciones interpersonales y un variado abanico de emociones, tanto positivas como negativas. Como Rodrigo Alsina (2001: 64) afirma, los sentimientos que surgen durante los encuentros interculturales también son influidos por los sistemas de creencias culturales y morales de una comunidad determinada. De ahí que los profesores de lenguas extranjeras, al desarrollar la competencia intercultural ${ }^{1}$ de sus alumnos, deban enseñarles también cómo sobrellevar emocionalmente las nuevas circunstancias sociales.

Uno de los factores que ayuda a entender mejor las diferencias culturales es la inteligencia emocional. Según Mayer y Salovey (1997: 5), esta es una habilidad que ayuda a percibir, acceder y generar emociones, así como también a facilitar el pensamiento. Por otra parte, es una capacidad que permite entender los sentimientos y regularlos de manera reflexiva. Se puede decir que también la actitud de apertura, respeto y tolerancia hacia las actuaciones de otras personas promueve un crecimiento emocional e intelectual. Goleman (1997: 162) añade que esta conciencia de uno mismo ayuda a desarrollar la empatía ${ }^{2}$, "puesto que, cuanto más abiertos nos hallemos a nuestras propias emociones, mayor será nuestra destreza en la comprensión de los sentimientos de los demás". El autor afirma también que la inteligencia emocional responde a la plasticidad cerebral, donde cualquier estímulo, práctica continuada y aprendizaje sistemático crea cambios positivos.

Otro concepto que, en nuestra opinión, juega un papel importante en la educación intercultural es la autoestima. Branden (2007: 21-22) la define como la confianza en uno mismo, en su mente, en sus capacidades para pensar, para enfrentarse a los desafíos, para ser feliz, etc. El nivel de autoestima puede afectar al "funcionamiento, motivación, comportamiento y adaptación eficaz del individuo a su medio

\footnotetext{
${ }^{1}$ La competencia intercultural forma parte de la competencia comunicativa (véase Spychała 2013) y se compone de tres elementos: los conocimientos (knowlegde), las habilidades (skills) y las actitudes (attitudes). Byram, Nichols y Stevens (2001: 4-7) explican que, aparte de tener los conocimientos acerca de otra cultura, el hablante intercultural debe saber cómo funcionan distintos grupos sociales, incluyendo sus identidades. Debido a que el profesor es incapaz de presentar y hablar en clase de todos los aspectos de las culturas de una lengua, el alumno debe tener las habilidades de buscar, interpretar, relacionar y comparar nuevos conocimientos culturales que consigue por su propia cuenta. Y las actitudes interculturales se basan en tener curiosidad y apertura durante un encuentro; se trata sobre todo de no analizar las diferencias desde el punto de vista de los propios valores, creencias o conductas, sino de aceptarlas y comprenderlas.

2 “La empatía es un factor, quizá el más importante, que contribuye a la coexistencia armoniosa de los individuos en la sociedad. Está íntimamente ligada a la relatividad cultural, que nos libera de nuestros condicionantes y nos ayuda a reconocer que nuestra forma de ser no es la única y posiblemente ni siquiera la mejor" (Arnold y Brown 2000: 36).
} 
ambiente" (González-Arratia López Fuentes, 2001: 13). Estudios como el de Gardner y MacIntyre (1993) confirman que en la clase de lengua extranjera un bajo nivel de autoestima puede ser provocado por una actitud negativa hacia la lengua estudiada, las calificaciones o incluso el choque cultural $^{3}$.

Sin embargo, según Oxford (2000), este tipo de ansiedad perjudicial puede ser momentánea, es decir, con el tiempo puede disminuir gracias a la actuación de los profesores de lenguas. Si un profesor quiere reducir la ansiedad, Oxford (2000: 85-86) sugiere algunas técnicas, como por ejemplo: ayudar a entender que las emociones negativas pueden ser transitorias, dejar muy claros los objetivos de la clase, dar permiso para cometer errores, reducir la competitividad, usar música, la risa y los juegos en clase con el fin de relajarse, animar a que hablen entre ellos de forma positiva. En este caso, la institución escolar influye también de manera significativa en el desarrollo de la autoestima: si el alumno se siente valorado a pesar de sus fracasos o los errores que comete, su autoestima y el estado de ánimo siguen en un nivel alto. En definitiva, se trata sobre todo de estimular las emociones positivas y preparar a los alumnos para gestionar los sentimientos negativos que puedan aparecer durante los encuentros interculturales.

\section{TRATAMIENTO DE LAS EMOCIONES EN LA CLASE DE ELE}

La psicóloga Annie de Acevedo (2005) recuerda que en los últimos años ha crecido el número de jóvenes con problemas de aprendizaje, también debido a diferentes desórdenes emocionales, lo que se debe entre otras cosas a la globalización, al desarrollo de las nuevas tecnologías, a los cambios climáticos o incluso a la mala alimentación. Basándonos en nuestra experiencia profesional como profesora de

\footnotetext{
${ }^{3}$ Según Hofstede (2000: 377), el choque cultural es "el estrés causado por encontrarse en un ambiente cultural ajeno al que pueden acompañar unos síntomas somáticos”. Oberg (1960: 177-178) describió cuatro fases del choque cultural o, mejor dicho, de la adaptación a otra cultura, e identificó varias emociones que puede experimentar una persona al pasar por una etapa determinada. Al principio, en las primeras semanas o meses de visita en un país nuevo, aparece la sensación de fascinación por lo nuevo. Es como si se estuviera de luna de miel. Todo cambia cuando hay que acudir a la escuela, al trabajo, a alquilar una casa, etc. En esta fase, que Oberg (1960) denomina etapa de crisis, uno empieza a sentirse frustrado, deprimido e incluso agresivo debido a la cantidad de dificultades y problemas que de repente se acumulan. Oberg (1960) añade que, si uno no sabe superarlo, vuelve a su país natal. Y si se queda, pasa a la fase de adaptación, en la que consigue ajustarse a la nueva cultura. Desde entonces está más animado, empieza a relacionarse más con otra gente, siente más confianza (por ejemplo, hablando en la lengua extranjera) y tiene más distancia respecto a sí mismo. Al final, no solo acepta la forma de vivir de los demás y sus "extrañas" costumbres, sino que también disfruta de las diferencias culturales. En esta fase de adaptación casi desaparece la sensación de ansiedad o la actitud etnocéntrica, es decir deja de analizar la otra cultura desde la perspectiva de la propia cultura, que ya no considera mejor que las demás.
} 
español de escuela secundaria, podemos constatar que el docente, al utilizar diversas estrategias de carácter afectivo, puede crear un ambiente acogedor, estable y seguro en la clase.

Una de las cosas que puede hacer el profesor para disminuir el estrés al principio de la clase es dejar de hacer evaluación oral frente a los demás alumnos. En realidad, cuando el alumno habla con un hispanohablante nunca experimentará este tipo de situación. Por eso, es mejor hablar con el alumno estando sentados, individualmente, cuando el resto de la clase está ocupado con otras tareas. Sin duda, un ambiente sano y no punitivo favorecerá la motivación y, especialmente, el equilibrio emocional de los alumnos.

Por otra parte, el alumno debería participar en la creación del programa y en la evaluación de sus resultados. De esta manera puede conseguir una buena relación con la materia y al mismo tiempo sentir "que la materia que se enseña le concierne a él personalmente, que él es activo en la determinación de las interconexiones que él tiene con el mundo. Que él no es solo un ente pasivo en la interconexión que otros han definido para él" (Ibarrolla 2015: 265).

Si el estudiante participa en la programación del currículo, el profesor también puede conocer sus intereses. Una de las tareas que influye en la motivación de los estudiantes es dejarles elegir un tema cultural de su predilección. El alumno, solo o en grupo, puede preparar una presentación u otro material que él mismo haya elegido (p. ej. el fútbol de Argentina, los animales en Perú, las fiestas de México, etc.). Si los alumnos se encuentran en un estadio básico de aprendizaje, lo pueden preparar en su lengua materna. Se recomienda hacerlo teniendo en cuenta el desarrollo de su sensibilidad o sensibilización cultural ${ }^{4}$. Por ejemplo, con la ayuda del profesor pueden hacer una comparación entre los conocimientos culturales de la cultura meta y su propia cultura.

Y, al introducir los entrenamientos interculturales ${ }^{5}$ (véase Spychała 2016), se trabaja la Inteligencia Emocional, generando al mismo tiempo conocimientos acerca

\footnotetext{
${ }^{4}$ Según el Marco común europeo de referencia (MCER 2002: 102), la sensibilidad cultural forma parte de las habilidades interculturales y se une con "la capacidad de identificar y utilizar una variedad de estrategias para establecer contacto con personas de otras culturas" Bennett y Bennett (2003: 155) han elaborado un interesante modelo de desarrollo de la sensibilidad intercultural (DMIS - The Developmental Model of Intercultural Sensitivity). Lo dividen en seis etapas: 1. Negación (no vemos las diferencias culturales), 2. Defensa (percibimos las diferencias, pero las evitamos), 3. Minimización de las diferencias (consideramos que las diferencias no son tan importantes), 4. Aceptación (empezamos a aceptar y respetar las diferencias culturales), 5. Adaptación (aprendemos nuevas costumbres, nos acercamos a otros valores y creencias a veces por encima de nuestra identidad), 6. Integración (nos integramos en la otra cultura al mismo tiempo que conservamos nuestra propia identidad). Podemos decir que este modelo de alguna manera se basa en las ya mencionadas etapas del choque cultural de Oberg (1960).

${ }^{5}$ El objetivo primordial de los entrenamientos interculturales es hacer que sus participantes conozcan mejor la otra cultura, a través de las interacciones interpersonales, que deben apoyarles
} 
de las diferencias culturales. Por ejemplo, el trabajo con los estereotipos es un buen método para desarrollar la empatía y la sensibilidad intercultural de los alumnos. La tarea consiste en una interacción grupal: cada persona (incluido el profesor) lleva pegada en su cabeza una nota en la que aparecen distintos nombres: español, andaluz, gallego, catalán, vasco, mexicano, argentino, etc. Su compañero tiene que decir qué le gusta o no le gusta en cuanto al lugar de procedencia de su compañero (España, Argentina, etc.), sin decir el nombre de este lugar. La persona que lleva la nota tiene que adivinar de dónde es. Luego, se puede organizar un debate o una discusión en torno a los conocimientos culturales (equivocados o no) sobre las diferentes naciones hispanohablantes teniendo en cuenta las emociones. Los alumnos, basándose en fuentes recomendadas por el profesor, también pueden preparar trabajos escritos sobre las naciones que les hayan tocado.

Al final, proponemos trabajar con la música, que casi siempre produce emociones positivas en la clase (sin mencionar la estimulación del hemisferio derecho). Las canciones son un recurso que aporta conocimientos lingüísticos, fonéticos y culturales (Siek-Piskozub, Wach 2006) ${ }^{6}$. A la hora de elegir una canción, hay que tener en cuenta la edad de los alumnos, su nivel de lengua, el tema y los objetivos comunicativos e interculturales. Por ejemplo, sugerimos empezar por la música popular de un país hispanohablante (Argentina, España, México, etc.). Los alumnos pueden, de esta forma, conocer la historia del país, la vida de sus habitantes, los instrumentos, distintos tipos de bailes o vestidos típicos. Además, si recurren a la cultura musical de su país nativo, desarrollan también su competencia intercultural.

\section{EL PAPEL DE LA AFECTIVIDAD EN EL CONTEXTO EDUCATIVO POLACO. RESULTADOS DE LA INVESTIGACIÓN}

En esta parte recurrimos al estudio que hicimos entre estudiantes de español en Polonia. Nuestra intención es mostrar que la enseñanza afectiva influye en las actitudes positivas hacia la lengua extranjera. Los resultados que presentamos forman parte de una investigación mayor, que ha sido realizada entre estudiantes de español como lengua extranjera en Polonia (véase Spychała-Wawrzyniak 2018). Los

\footnotetext{
y facilitarles el futuro contacto con representantes de otras comunidades (Brisilin y Yoshida 1994; Atroszko 2008).

${ }^{6}$ Vale la pena mencionar que, entre las razones para elegir el español, los estudiantes polacos señalaron en primer lugar que les gusta el sonido de la lengua española y en cuarto lugar, que les gusta escuchar música en español. En segundo y tercer lugar los alumnos indicaron que habían elegido el español porque es la segunda lengua más usada en el mundo y porque no querían estudiar alemán (Spychała-Wawrzyniak 2018).
} 
datos fueron recogidos en el año escolar 2015-2016, a partir de un cuestionario (en formato electrónico) cuyo objetivo primordial era analizar las actitudes hacia el aprendizaje de ELE por parte de los alumnos que habían participado en las Olimpiadas Nacionales de español. Se analizaron 782 encuestas de 622 alumnas (79,5\%) y 160 alumnos (20,5\%). La mayoría de ellos $(612-78,13 \%)$ estudiaba en una ciudad de más de 50000 habitantes. Muchos de ellos eran estudiantes de liceum $^{7}$ (662 alumnos - 84,7\%); los demás (120 alumnos - 15,4\%) representaban otros niveles de enseñanza (gimnazjum, technikum). El 98,8\% de los encuestados aprendía español en la escuela, de los cuales el $62,8 \%$ declaró que tenía entre dos y tres horas semanales de español.

En este espacio nos centramos en las respuestas a dos preguntas abiertas: 1) ¿Qué tipo de acciones realizadas por el profesor les gustan a los alumnos?, 2) ¿Qué es lo que no les gusta a los alumnos en la clase de ELE? Los encuestados respondieron en polaco de forma libre. Para cada pregunta recibimos más de 700 opiniones, que han sido categorizadas según el tipo de repuesta. Debemos mencionar que los alumnos evaluaron también las competencias de sus profesores. El 90\% de los alumnos otorgaron a sus profesores una nota excelente, muy buena o buena. En cuanto a sus opiniones sobre los docentes, podemos decir que la mayoría de los alumnos se centró en aspectos cognitivos (67\% de las respuestas): enseña bien o mal, conoce bien la lengua, tiene buen conocimiento cultural, tiene experiencia, es competente, etc. En el siguiente lugar se encuentran las opiniones (33\%) relacionadas con los factores afectivos (estilo motivador, buena actitud, comprensivo, etc. $)^{8}$. Podemos concluir que, sorprendentemente, lo que domina en las evaluaciones de los alumnos acerca de las competencias docentes, es el factor cognitivo.

En cuanto a las valoraciones de lo que les gusta o no les gusta hacer a los alumnos en la clase de español, el estudio claramente demuestra que prefieren la diversidad en las acciones realizadas por el profesor (fig. 1). Entre ellas enumeran: las anécdotas culturales, las conversaciones en español, las canciones, las películas, los juegos, etc.

En la siguiente figura (fig. 2) presentamos los elementos que no les agradan a los alumnos en la clase de español. Los resultados muestran que la mayoría de los alumnos rechaza las actividades de carácter sumamente cognitivo: no quieren estudiar gramática, hacer exámenes, escuchar audiciones (excepto las canciones), hablar en polaco, etc.

\footnotetext{
${ }^{7}$ El sistema educativo polaco está formado por las escuelas de enseñanza primaria y secundaria básica (gimnazjum) y superior (liceum, technikum). Debido a la reforma del sistema educativo en el año 2017 se ha empezado a liquidar los centros de enseñanza media (gimnazjum) para volver al sistema de ocho años de educación primaria y cuatro de secundaria.

${ }^{8}$ Hay que añadir que en la suma total no hemos contado las respuestas negativas, tipo: no exige, no controla ni domina la clase, etc.
} 


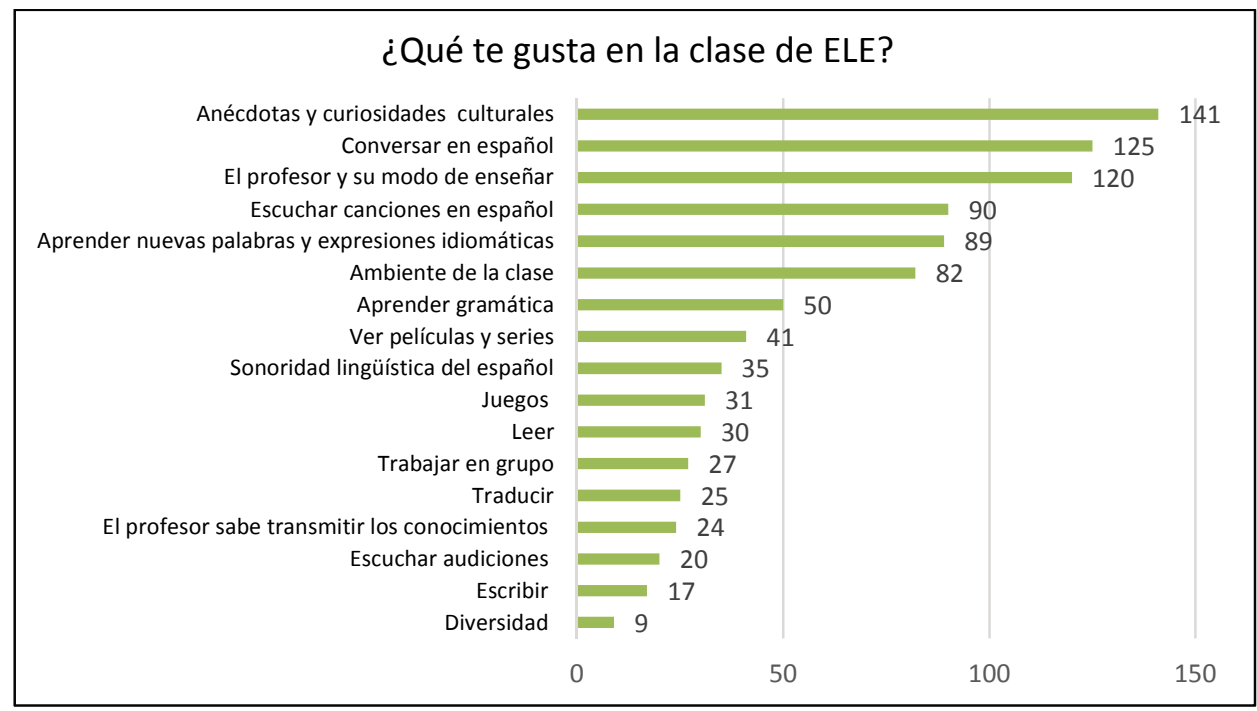

Fig. 1. Análisis de las acciones que les gustan a los alumnos en la clase de ELE en Polonia (Elaboración propia a partir de Spychała-Wawrzyniak 2018: 146)

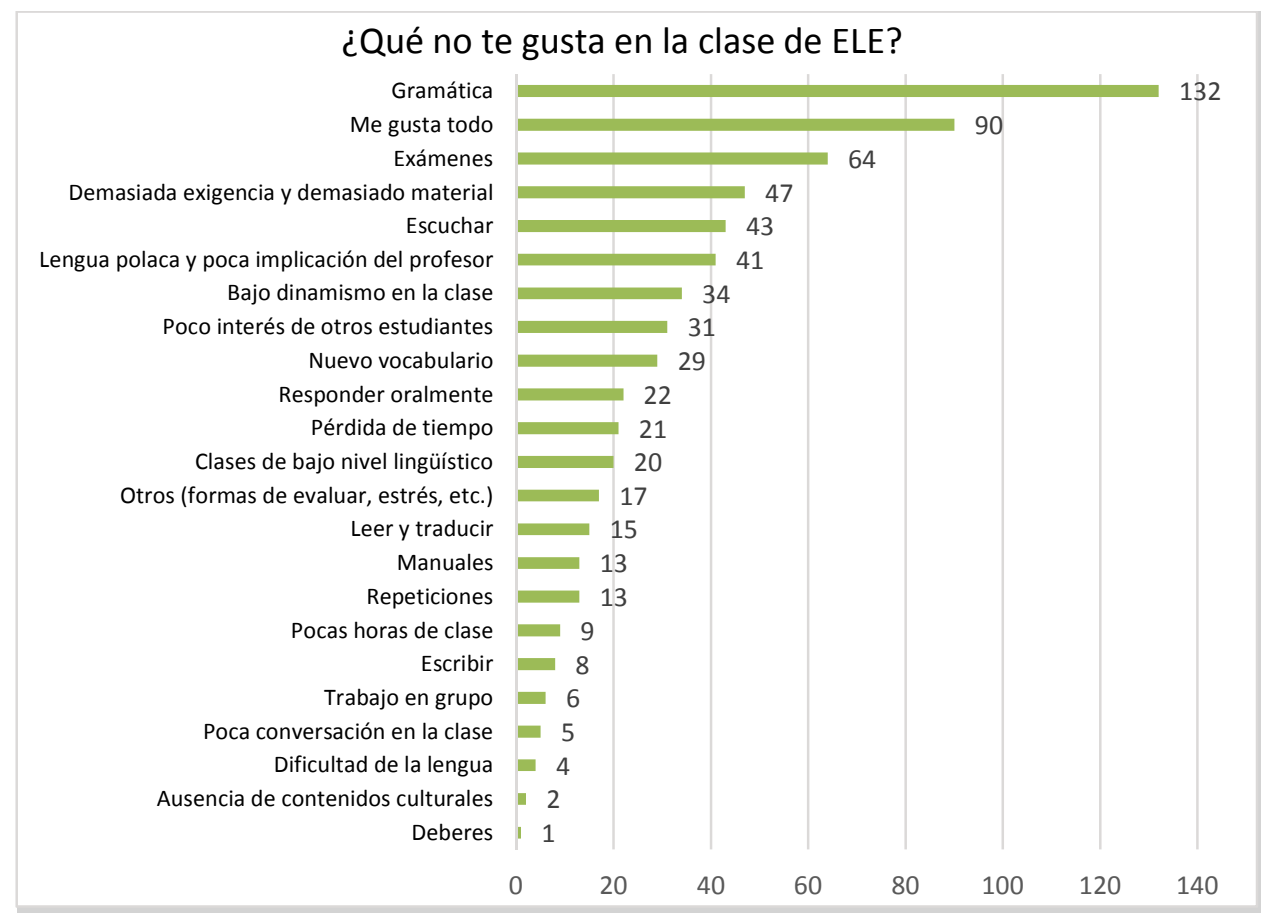

Fig. 2. Análisis de las acciones que no les gustan a los alumnos en la clase de ELE en Polonia (Elaboración propia a base de Spychała-Wawrzyniak 2018: 154) 
Resumiendo, podemos confirmar que el entorno afectivo ejerce una influencia bastante importante en la enseñanza de español en las escuelas polacas. Se espera que el profesor funcione como animador y que utilice diferentes técnicas estimulantes. Hay que subrayar también que los alumnos de español agradecen cualquier información sobre las culturas hispanohablantes. Si el profesor lo tiene en cuenta, puede llegar a ser un buen profesor de español, ya que el mismo estudio (SpychałaWawrzyniak 2018) demostró que, según los estudiantes que aprenden español en las escuelas polacas, el profesor ideal en primer lugar debería ser inteligente, competente y saber muy bien la lengua española (lo que constituyó el $25,60 \%$ de las respuestas). Sin embargo, en los siguientes puestos aparecen características típicamente afectivas: carismático y apasionado $(25,40 \%)$, paciente y tranquilo $(24,40 \%)$, amable y de confianza $(23,90 \%)$. Estas valoraciones claramente demuestran que el profesor a la hora de dictar la clase debe tratar con especial atención la dimensión afectiva, porque es esto lo que esperan de él los alumnos.

\section{CONCLUSIONES}

El proceso de globalización y el fácil acceso a otras comunidades culturales han dejado su huella en la enseñanza de lenguas extranjeras. Se nota que cada día esta disciplina evidencia una transformación en métodos y técnicas (también gracias el desarrollo de las nuevas tecnologías). Aunque el sistema educativo en muchos casos mantiene sus estructuras tradicionales, los profesores tienen que aceptar los nuevos cambios, incluido el hecho de que cambian sus alumnos, y adaptarse a ellos. No cabe duda que el saber emocional del docente puede apoyarle en comprender mejor las emociones de sus estudiantes. Como dice Ibarrolla (2015: 227), "las emociones nos proporcionan un cerebro químicamente estimulado y más activado, que nos ayuda a recordar mejor las cosas". Si el profesor conoce los intereses de sus alumnos, puede subir su autoestima dando más sentido a lo que están haciendo. Es aconsejable que el material (también cultural) resulte atractivo e interesante para ellos. De este modo, el desarrollo de la competencia comunicativa intercultural en el entorno escolar será más eficaz. Recordemos, por último, que según Maqueo (2005), en la clase de lengua extranjera, la cultura del alumno y la cultura de los países de la lengua aprendida también influyen en el manejo de las emociones del estudiante, lo que vienen a confirmar, a su vez, los resultados de la investigación realizada entre los estudiantes de ELE en Polonia. 


\section{BIBLIOGRAFÍA}

Acevedo, A. (2005). Casos y cosas. La realidad de los niños y los jóvenes de hoy. Bogotá: Grupo Editorial Norma.

Arnold, J., Brown, H.D. (2000). Mapa del terreno. In J. Arnold (ed.), La dimensión afectiva en el aprendizaje de idiomas (pp.19-41). Madrid: Cambridge University Press (original work published 1999). Trad. A. Valero.

Atroszko, P. (2008). Trening kompetencji kulturowej. Technika incydentu krytycznego i asymilatora kulturowego. In J. Rajang y J. Lessing-Pernak (eds.), Psychologia w życiu osobistym i zawodowym człowieka (pp. 111-123). Bydgoszcz: Wydawnictwo Uniwersytetu Kazimierza Wielkiego.

Bennett, J.M., Bennett, M.J. (2003). Developing intercultural sensitivity. An integrative approach to global. And domestic diversity. In D. Landis, J.M. Bennett y M.J. Bennett (eds.), Handbook of intercultural training (3rd edition) (pp. 147-165). Thousand Oaks: SAGE.

Branden, N. (2007). Los seis pilares de la autoestima. Madrid: Paidós (original work published in 1994). Trad. J. Vigil Rubio.

Brislin, R., Yoshida, T. (1994). Intercultural communication training. An introduction. Thousand Oaks, CA: SAGE Publications.

Byram, M., Nichols, A., Stevens, D. (2001). Introduction. In M. Byram, A. Nichols, D. Stevens (eds.), Developing Intercultural competence in Practice (pp. 1-8). Clevedon: Multilingual Matters.

Gardner, R.C., MacIntyre, P.D. (1993). On the measurement of affective variables in second language learning. Language Learning, 43, 157-194.

Goleman, D. (1997). Inteligencia emocional. Barcelona: Kairós (original work published in 1995). Trad. D. González Raga y F. Mora.

González-Arratia López Fuentes, N.I. (2001). La autoestima. Mediación y estrategias de intervención a través de una experiencia en la reconstrucción del ser. Toluca: Universidad Autónoma del Estado de México.

Hofstede, G. (2000). Kultury i organizacje. Warszawa: Polskie Wydawnictwo Ekonomiczne (original work published in 1997). Trad. M. Durska.

Ibarrolla, B. (2015). Aprendizaje emocionante. Neurociencia para el aula. Madrid: SM.

Maqueo, A.M. (2005). Lengua, aprendizaje y enseñanza. El enfoque comunicativo: de la teoría a la práctica. México D.F.: Editorial Limusa.

Mayer, J.D., Salovey, P. (1997). What is Emotional Intelligence? In P. Salovey y D.J. Sluyter (eds.), Emotional development and emotional intelligence educational implications (pp. 3-31). New York: Basic Books.

MCER, Consejo de Europa (2002). Marco común europeo de referencia para las lenguas: aprendizaje, enseñanza, evaluación. Madrid: Secretaría General Técnica del MEC, Anaya e Instituto Cervantes (original work published in 2001), https://cvc.cervantes.es/ensenanza/ biblioteca_ele/marco/cvc_mer.pdf [02.01.2019].

Oberg, K. (1960). Culture shock: adjustment to new cultural environments. Practical Anthropology, 7, 177-182.

Oxford, R.L. (2000). La ansiedad y el alumno de idiomas: nuevas ideas (pp. 77-86). In J. Arnold (ed.), La dimensión afectiva en el aprendizaje de idiomas (pp. 19-41). Madrid: Cambridge University Press (original work published in 1999). Trad. A. Valero. 
Rodrigo Alsina, M. (2001). Teorías de la comunicación: ámbitos, métodos y perspectivas. Barcelona: Universitat Autònoma de Barcelona.

Siek-Piskozub, T., Wach, A. (2006). Muzyka i stowa: rola piosenki $w$ procesie przyswajania języka obcego. Poznań: Wydawnictwo Naukowe UAM.

Spychała, M. (2013). Ewolucja struktury kompetencji komunikacyjnej: od kompetencji językowej do interkulturowej kompetencji negocjacyjnej. In J. Stańczyk, E. Nowikiewicz (eds.), Dydaktyka językowa a kompetencje ogólne (pp. 25-34). Bydgoszcz: Nauczycielskie Kolegium Języków Obcych.

Spychała, M. (2016). Trening interkulturowy podczas lekcji języka obcego na przykładzie języka hiszpańskiego. In A. Jaroszewska, B. Karpeta-Peć, M. Smuk, J. Sobańska y J. Sujecka-Zając (eds.), Wielojęzyczność i międzykulturowość na lekcji języka obcego (pp. 77-93). Warszawa: Uniwersytet Warszawski.

Spychała-Wawrzyniak, M. (2018). Las actitudes de los alumnos activos hacia el aprendizaje del español como lengua extranjera (ELE). Una visión constructivista. Poznań: Wydawnictwo Naukowe UAM. 